\title{
Influência das Variáveis do Treinamento Contra-Resistência Sobre o Consumo de Oxigênio em Excesso Após o Exercício: Uma Revisão Sistemática
}

\author{
Influence of Resistance Training Variables on Post-Exercise Oxygen \\ Consumption: a Systematic Review
}

Antonio Gil Castinheiras Neto ${ }^{1}$ Nádia Lima da Silva

Paulo de Tarso Veras Farinatti ${ }^{1-2}$

1. Programa de Pós-Graduação em Ciências da Atividade Física - Universidade Salgado de Oliveira - 24030-060 - Niterói. Brasil.

2. Laboratório de Atividade Física e Promoção da Saúde - Universidade do Estado do Rio de Janeiro

- 20550-900 - Rio de Janeiro. Brasil.

Endereço para correspondência: Paulo T.V. Farinatti, Programa de Pós-Graduação em Ciências da Atividade Física, Universidade Salgado de Oliveira, Rua Marechal Deodoro, 211, Bloco C, 10 andar - 24030-060 - Niterói.

E-mail:pfarinatti@gmail.com

Submetido em 04/05/2008

Versão final recebida em 17/10/2008 Aceito em 28/11/2008

\begin{abstract}
RESUMO
O exercício contra-resistência (ECR) pode contribuir para o aumento da taxa metabólica de repouso (TMR). É consenso na literatura que o volume da sessão de ECR pode repercutir em maior gasto calórico e que, após o exercício, o consumo de oxigênio em excesso (EPOC) pode variar de acordo com a característica do programa de exercício. Contudo, ainda não é possível definir qual ou quais variáveis de prescrição têm maior impacto sobre o EPOC em sessões de ECR. O objetivo do estudo foi efetuar uma revisão sistemática sobre os estudos que se propuseram a investigar as relações entre o EPOC e variáveis de treinamento em ECR. Os 17 estudos selecionados foram agrupados por similaridade de tratamento (número de séries, intensidade, intervalos de recuperação, velocidade da contração muscular e ordem dos exercícios). Técnicas de estatística descritiva foram utilizadas na tentativa de estabelecer possíveis tendências nas relações dose-resposta. Posteriormente, os dados foram analisados de forma qualitativa. De todas as variáveis analisadas, o curto intervalo de recuperação e o modo de prescrição no formato em circuito foram aquelas com maior impacto provável sobre a magnitude do EPOC. Quanto às demais variáveis, limitações metodológicas não permitem estabelecer tendências, principalmente no tocante à duração do EPOC. Adicionalmente, constata-se a necessidade de padronização de aspectos importantes para a quantificação do EPOC, como o tempo de observação do após o exercício e a forma de aferição da TMR.
\end{abstract}

Palavras-chave: EPOC, fisiologia do exercício, treinamento de força, aptidão física, saúde.

\begin{abstract}
Resistance training (RT) may influence resting metabolic rate (RMR) increase. There is a consensus that the volume of the RT session may produce higher caloric expenditure and that the excess post-exercise oxygen consumption (EPOC) may vary according to the characteristics of the exercise program. However, it is not possible yet to define which prescription variables have greater impact on the EPOC. The aim of the present study was to systematically review the studies that investigated the relationship between EPOC and training variables. A total of 17 studies were selected, being organized according to treatment similarity (number of sets, intensity, rest interval, speed of muscle contraction and exercise order). Descriptive statistical techniques were used to establish possible tendency in dose-response relationships. Subsequently, data were qualitatively analyzed. The available evidence suggested that a short rest interval and the circuit training prescription mode had the greatest impact on the EPOC magnitude. As for the other variables, it was not possible to establish any tendency due to methodological limitations, especially concerning EPOC duration. In addition, it seems to be necessary to standardize important aspects of the EPOC assessing, such as the period of observation after exercise and criteria for measuring RMR.
\end{abstract}

Keywords: EPOC, exercise physiology, resistance training, physical fitness, health.

\section{INTRODUÇÃO}

Do ponto de vista epidemiológico, o gasto energético diário representa uma das maiores preocupações para a obesidade e demais doenças crônico-degenerativas. Nesse sentido, em posicionamento oficial conjunto, o American College of Sports and Medicine e a American Heart Association ${ }^{(1)}$ sugeriram que o gasto energético proveniente da realização de exercício físico deveria situar-se entre 1.000 e 2.000kcal/ semana. O exercício aeróbio, por possibilitar trabalhar grandes grupamentos musculares de forma contínua, é indicado por diversas agências normativas da área da saúde para que se atinja esse montante ${ }^{(2-5)}$.
Ao contrário dos exercícios aeróbios, a contribuição do exercício contra-resistência (ECR) para a perda de peso é ainda bastante questionável, sobretudo pelo baixo gasto energético que parece propiciar ${ }^{(6,7)}$. Enquanto alguns autores relataram que sua maior contribuição seria derivada do próprio custo da execução dos exercícios (sessão de treinamento)(8), outras evidências sugerem que benefícios poderiam advir do aumento da taxa metabólica de repouso (TMR), com impacto de longo prazo na composição corporal ${ }^{(9)}$.

É aceito que, após uma sessão de ECR, a TMR permaneça elevada. Assim, nas últimas décadas alguns estudos buscaram esclarecer a contribuição do ECR para o gasto calórico diário em geral e, em particular, 
as características do consumo de oxigênio em excesso após o exercício (EPOC). No entanto, o EPOC é uma variável cujo comportamento em programas de ECR necessita ser mais bem investigada, haja vista que muitas dúvidas ainda permanecem sobre o tema, considerando o somatório de sessões de ECR isoladas ou combinadas com exercícios aeróbios. Um dos aspectos consensuais refere-se à aceitação de que o EPOC decorre de uma combinação de diversas variáveis do treinamento, como o número de séries, intensidade dos exercícios, tempo de intervalo de recuperação entre séries e exercícios, método de treinamento, velocidade de execução do movimento ou ordem dos exercícios.

As diferentes combinações dessas variáveis podem influenciar tanto a magnitude quanto a duração do EPOC, em seus componentes rápido, lento e ultra-lento ${ }^{(10)}$. Para Gaesser e Brooks ${ }^{(11)}$, a base metabólica do EPOC pode ser analisada a partir dos fatores que a influenciam, como os níveis de catecolaminas, tiroxinas, glicocorticóides, metabolismo de ácidos graxos e temperatura corporal. No componente rápido, o reabastecimento dos estoques de oxiemoglobina e oximioglobina, a restauração dos fosfagênios e a energia necessária para a reconversão do lactato em glicogênio explicariam até metade do EPOC em sua fase rápida ${ }^{(12)}$. Fatores como o aumento da temperatura corporal, a manutenção de circulação hipercinética ou a ventilação elevada também poderiam ter relação com maior consumo de oxigênio na primeira hora subseqüente a uma sessão de treinamento com ECR ${ }^{(13)}$. Além disso, associam-se ao componente lento do EPOC o metabolismo dos ácidos graxos, maior catecolanemia, presença do cortisol e dano muscular reativo a estratégias de treinamento, dentre outras variáveis que podem justificar EPOC aumentado por vários dias ${ }^{(11,14)}$.

Constata-se, então, que há mais discordância do que consenso entre os estudos disponíveis. Alguns deles sustentam que, mesmo após sessões extenuantes de ECR, o EPOC não seria significativo para um gasto calórico diário importante ${ }^{(15)}$. Outros, porém, indicam que a TMR pode permanecer aumentada em 20\% por até 39 horas $^{(9)}$. A grande disparidade metodológica entre os estudos parece ser a principal razão de resultados tão divergentes, principalmente quanto aos critérios para a quantificação de elementos importantes para a definição do gasto calórico.

Autores como Murphy e Schwarzkop ${ }^{(16)}$, por exemplo, aferiram a taxa metabólica de repouso em 5 min, enquanto outros como Ratamess et al. ${ }^{(17)}$ fizeram-no em 30 min. O critério adotado para estabelecer o dispêndio energético proveniente do ECR também não é padronizado: alguns relatam o gasto calórico total da sessão, que inclui a mensuração do consumo de oxigênio durante o exercício somado ao $\mathrm{EPOC}^{(17-25)}$, outros apenas mensuram o $\mathrm{EPOC}^{(9,14,15,26-28)}$ ou nem isso, não considerando o período de recuperação pós-exercício para o cálculo do gasto calórico total da sessão ${ }^{(8,29)}$. Dentre os métodos de prescrição do treinamento, foram testadas as influências de trabalhos em circuito e em séries consecutivas ${ }^{(16,19,22)}$, manipulando-se o número de séries ${ }^{(23,24)}$, a intensidade $\mathrm{e}^{(16,17,20,26,27)}$, o intervalo de recuperação entre séries e exercícios ${ }^{(17,19)}$, a velocidade da contração muscular ${ }^{(14,20,21)}$ e a ordem dos exercícios ${ }^{(15)}$.

Em virtude dessas diferenças, também os resultados dos estudos disponíveis apresentam variações para os EPOCs obtidos: a amplitude de variação da magnitude do EPOC em ECR nos estudos localizados oscilou entre $\approx 5,5 \mathrm{kcal}^{(26)} \mathrm{e} \approx 775 \mathrm{kcal}^{(9)}$, com mediana de $41 \mathrm{kcal}$; a duração do EPOC variou entre 15 min $^{(20)}$ e 48h ${ }^{(14)}$. Uma duração acima de 1h para o EPOC foi encontrada em quatro estudos ${ }^{(9,14,23,24)}$, sendo em dois deles superior a $24 h^{(9,14)}$. Três estudos encontraram EPOC significa-

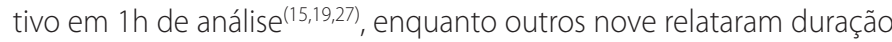
inferior a $1 \mathrm{~h}^{(16,17,20-22,25-27,30)}$, sendo 30 min o valor modal. Um estudo, enfim, não mensurou a cinética do EPOC, não divulgando sua duração e limitando-se em confrontar os valores do somatório entre o $\mathrm{VO}_{2}$ da sessão e o EPOC sobre o $\mathrm{VO}_{2}$ estimado do grupo controle ${ }^{(18)}$. O período de observação do EPOC, por sua vez, também varia muito, indo de 20 $\min ^{(16,25)}$ a $72 \mathrm{~h}^{(14)}$. O intervalo entre as medidas durante o EPOC varia de $5 \min ^{(17)}$ a $24 h^{(14)}$. Outro ponto a se destacar é que, dentre os 17 estudos que investigaram a cinética do EPOC após sessão de ECR, 11 iniciaram a medida imediatamente após o término do exercício.

Permanecem, portanto, dúvidas sobre a possível influência das variáveis do ECR sobre o EPOC. De fato, ainda não é possível indicar qual estratégia de ECR produziria maior magnitude e/ou duração de um gasto calórico superior ao do repouso, o que seria evidentemente interessante no contexto de programas de emagrecimento. Por outro lado, há carência de estudos que se tenham proposto a analisar conjuntamente os dados disponíveis no que diz respeito aos fatores determinantes do EPOC decorrente de sessões de ECR. Assim, o objetivo do trabalho foi efetuar uma revisão sistemática dos estudos sobre as relações entre o EPOC e as variáveis do ECR (número de séries, carga, intervalos de recuperação, modo de treinamento, velocidade de execução e ordem dos exercícios).

\section{MÉTODOS}

Foi conduzida uma revisão sistemática com base nos seguintes critérios de inclusão de estudos: a) estudos experimentais cujos tratamentos envolviam exclusivamente treinamento de força e tinham como objetivo a análise do EPOC; b) amostras compostas por indivíduos adultos saudáveis e jovens de ambos os sexos. Foram analisados estudos publicados e encontrados através de busca eletrônica no Medline, Lilacs e Sport Discus. Foram utilizados algoritmos de busca aceitos pela literatura especializada, a listagem de referências e busca manual dos artigos em revistas não localizadas por meio virtual. Na seleção inicial foram filtrados 24 estudos, dos quais apenas 17 atenderam aos critérios para inclusão. A partir de então, foram analisados separadamente os resultados obtidos, bem como suas possíveis relações com as seguintes variáveis: número de séries, intensidade da carga, intervalos de recuperação, modo de treinamento, velocidade de execução e ordem dos exercícios.

A maioria dos estudos sobre o EPOC em ECR testou dois ou mais métodos de prescrição objetivando comparações intergrupos. Os resultados encontrados foram agrupados por similaridade de tratamento e respectivo efeito sobre o EPOC (magnitude e duração). Em alguns casos, foi necessário reconverter os dados absolutos relatados (de kilojoule para quilocaloria) ou subtrair os dados brutos do $\mathrm{VO}_{2}$ da sessão e do EPOC pelo gasto energético em repouso, em uma mesma duração, a fim de obter o gasto calórico líquido da sessão ou o Net Caloric Coust. Ainda, quando a magnitude do EPOC não era relatada, a fim de estimá-la multiplicaram-se os valores de $\mathrm{VO}_{2}$ pós-exercício que excediam a TMR pelo tempo de duração do EPOC.

Utilizou-se a estatística descritiva para apreciar as relações doseresposta dos tratamentos. A aplicação da mediana como medida de tendência central foi justificada por ser menos sensível a valores extremos do que a média aritmética, já que resultados muito díspares foram visualizados na tabulação dos resultados dos estudos. As diferenças dessas respostas foram analisadas qualitativamente.

\section{RESULTADOS E DISCUSSÃO}

A tabela 1 apresenta as características gerais dos 17 estudos selecionados para a análise (amostra, características dos protocolos de treinamento e resultados obtidos para a magnitude e duração do EPOC). As próximas sessões analisam, de forma separada, os prováveis efeitos de cada uma das variáveis de treinamento (número de séries, velocidade de execução, intensidade da carga, intervalo de recuperação, método de treinamento e ordem dos exercícios). 
Tabela 1. Amostra, sessão de treinamento, resultados de estudos que investigaram o EPOC em ECR e observações

\begin{tabular}{|c|c|c|c|c|c|c|c|c|}
\hline Estudo & Amostra & Sessão de ECR & $\begin{array}{l}\text { Variáveis } \\
\text { observadas }\end{array}$ & $\begin{array}{l}\text { Tempo de } \\
\text { medida } \\
\text { EPOC }\end{array}$ & $\begin{array}{l}\text { Duração } \\
\text { do EPOC }\end{array}$ & $\begin{array}{l}\text { Magnitude do } \\
\text { EPOC }\end{array}$ & Observações & $\begin{array}{c}\text { Justificativa para o } \\
\text { tempo de medida } \\
\text { proposto para o EPOC }\end{array}$ \\
\hline Elliot et al. (22) & $\begin{array}{c}4 \mathrm{H}-\mathrm{T} \text { e } 5 \mathrm{M}-\mathrm{T} \\
(25,5)\end{array}$ & $\begin{array}{c}4 \text { séries (C) de } 15 \text { rep a 50\% 1RM, int. } \\
30 \text { s e } 3 \text { séries ( } 5 \text { ) } 5 \text { rep a 85\% 1RM, int. } \\
\text { 90s (8 exercícios) }\end{array}$ & TMR e EPOC & $90 \mathrm{~min}$ & $30 \mathrm{~min}$ & $\begin{array}{l}49 \pm 2 \mathrm{kcal} \mathrm{e} \\
51 \pm 3 \mathrm{kcal}\end{array}$ & Nenhuma & ND \\
\hline $\begin{array}{c}\text { Murphy e } \\
\text { Schwarzkopf }{ }^{(16)}\end{array}$ & $\begin{array}{l}10 M-T \\
(22 \pm 2)\end{array}$ & $\begin{array}{c}3 \text { séries (C) 50\% de 1RM, int. } 30 \text { s e } \\
3 \text { séries(S) 80\% de 1RM, int. } 2 \text { min (6 } \\
\text { exercícios) }\end{array}$ & TMR e EPOC & $20 \mathrm{~min}$ & $20 \mathrm{~min}$ & $\begin{array}{l}25 \mathrm{kcal} \mathrm{e} \\
13,5 \mathrm{kcal}\end{array}$ & TMR medida por 5 min. & ND \\
\hline Melby et al..(23) & $7 \mathrm{H}-\mathrm{T}(30 \pm 8)$ & $\begin{array}{c}6 \text { séries (S) 70\%1RM, int. } 3 \text { min e } 5 \\
\text { séries (S) 70\%1RM, int. } 4 \text { min (10 } \\
\text { exercícios) }\end{array}$ & $\begin{array}{l}\text { TMR, RER e } \\
\text { EPOC }\end{array}$ & $15 \mathrm{~h}$ & $15 \mathrm{~h}$ & $\begin{array}{c}15 \mathrm{~h}=113 \mathrm{kcal} \mathrm{e} \\
114 \mathrm{kcal}\end{array}$ & $\begin{array}{l}\text { Só iniciou a medida do } \\
\text { EPOC após o } 5^{\circ} \mathrm{min}\end{array}$ & $\begin{array}{l}\text { Investigar oxidação } \\
\text { lipídica após ECR } \\
\text { extenuante. }\end{array}$ \\
\hline $\begin{array}{c}\text { Olds e } \\
\text { Abenerthy }\end{array}$ & $\begin{array}{c}7 H-\mathrm{T} \\
(34 \pm 14)\end{array}$ & $\begin{array}{c}2 \text { séries (S) 75\% 1RM, int. 3,5 min e } \\
2 \text { séries (S) 60\% 1RM, int. 3,5 min (7 } \\
\text { exercícios) }\end{array}$ & $\begin{array}{l}\text { TMR, FC, } \\
\text { temperatura } \\
\text { corp, RER e } \\
\text { EPOC }\end{array}$ & $5 \mathrm{~h}$ & $60 \mathrm{~min}$ & $\begin{array}{c}41,1 \pm 4 \mathrm{kcal} \mathrm{e} \\
32,5 \pm 3 \mathrm{kcal}\end{array}$ & $\begin{array}{l}\text { Durante o EPOC não } \\
\text { houve repouso total } \\
\text { entre as medidas; } \\
\text {-Amostra heterogênea. }\end{array}$ & ND \\
\hline Haltom et al. ${ }^{(19)}$ & $7 \mathrm{H}-\mathrm{T}(27 \pm 1)$ & \begin{tabular}{|c|}
2 séries (C) de 20 rep a $75 \%$ de $20 \mathrm{RM}$, \\
int. 20 s e 2 séries (C) de 20 rep a $75 \%$ \\
de $20 \mathrm{RM}$, int. 60 s (8 exercícios)
\end{tabular} & $\begin{array}{l}\text { TMR, } \mathrm{VO}_{2} \text { e } \\
\text { EPOC }\end{array}$ & $60 \mathrm{~min}$ & $60 \mathrm{~min}$ & $\begin{array}{l}51 \pm 2 \mathrm{kcal} \mathrm{e} \\
37 \pm 2 \mathrm{kcal}\end{array}$ & \begin{tabular}{|c|}
$\begin{array}{c}\text { Tempo de trabalho } \\
\text { muito discrepante entre } \\
\text { os experimentos }\end{array}$ \\
\end{tabular} & $\begin{array}{c}\text { Em estudo-piloto foi } \\
\text { determinado o período } \\
\text { de análise do EPOC }\end{array}$ \\
\hline Dolezal et al. ${ }^{(14)}$ & $\begin{array}{c}9 \mathrm{H}-\mathrm{NT} \\
(20,7 \pm 2,1)\end{array}$ & $\begin{array}{c}8 \text { séries (S) 6RM, int. } 3 \text { min, } 4 \text { s exc. (1 } \\
\text { exercício) }\end{array}$ & $\begin{array}{l}\text { TMR, EPOC, } \\
\text { CPK e PSE }\end{array}$ & $72 \mathrm{~h}$ & $48 \mathrm{~h}$ & $768 \mathrm{kcal}$ & $\begin{array}{l}\text {-Apenas } 1 \text { exercício; } \\
\text {-EPOC: dados brutos } \\
\text { reconvertidos }\end{array}$ & $\begin{array}{c}\text { Hipótese de TMR } \\
\text { aumentada por } \\
\text { ECR com ênfase em } \\
\text { componente excêntrico }\end{array}$ \\
\hline Binzen et al..(15) & $\begin{array}{l}10 M-T \\
(29 \pm 3) \\
\end{array}$ & $\begin{array}{l}3 \text { séries (S) 10RM, int. } 1 \text { min, } 2 \mathrm{~s} \\
\text { conc./4s exc. (10 exercícios) }\end{array}$ & \begin{tabular}{|c|} 
TMR, RER, La e \\
EPOC
\end{tabular} & $120 \mathrm{~min}$ & $60 \mathrm{~min}$ & $31 \mathrm{kcal}$ & Nenhuma & $\begin{array}{l}\text { Avaliar o impacto do } \\
\text { ECR na oxidação lipídica }\end{array}$ \\
\hline $\begin{array}{l}\text { Thornton e } \\
\text { Potteiger (26) }\end{array}$ & $\begin{array}{l}14 M-T \\
(27 \pm 5)\end{array}$ & $\begin{array}{l}2 \text { séries (S) de } 8 \text { rep a } 85 \% \text { 1RM, int. } \\
1 \text { min e } 2 \text { séries (S) de } 15 \text { rep a } 45 \% \\
\text { 1RM, int. } 1 \text { min (9 exercícios) }\end{array}$ & $\begin{array}{l}\text { TMR, VE, FC, } \\
\text { RER, La e } \\
\text { EPOC }\end{array}$ & $120 \mathrm{~min}$ & $20 \mathrm{~min}$ & $\begin{array}{c}11,0 \pm 1,9 \mathrm{kcal} \mathrm{e} \\
5,5 \pm 1,3\end{array}$ & $\begin{array}{l}\text {-ND duração do EPOC. } \\
\text {-O valor apresentado } \\
\text { trata-se de estimativa } \\
\text { visual gráfica ND }\end{array}$ & ND \\
\hline Schuenke et al. ${ }^{(9)}$ & $7 \mathrm{H}-\mathrm{T}(22 \pm 3)$ & $\begin{array}{l}4 \text { séries (C) 10RM, int. } 2 \text { min (3 } \\
\text { exercícios) }\end{array}$ & TMR e EPOC & $4 \mathrm{~h}$ & $3 \mathrm{~h}$ & $773 \mathrm{kcal}$ & \begin{tabular}{|c|} 
- EPOC: dados brutos \\
reconvertidos; \\
-Não controlaram fatores \\
de influencia da TMR \\
após ECR \\
\end{tabular} & $\begin{array}{l}\text { Investigar o distúrbio } \\
\text { causado por protocolo } \\
\text { de ECR intenso sobre } \\
\text { EPOC }\end{array}$ \\
\hline Hunter et al. ${ }^{(20)}$ & 7H-T (24 \pm 3$)$ & $\begin{array}{c}2 \text { séries }(\mathrm{S}) \text { de } 8 \text { rep a } 65 \% 1 \mathrm{RM}, 1 \mathrm{~s} \\
\text { conc./1 s exc., int. } 1 \text { min e } \\
2 \text { séries }(\mathrm{S}) \text { de } 8 \text { rep a } 25 \% 1 \mathrm{RM}, 10 \mathrm{~s} \\
\text { conc./5s exc., int. } 1 \text { min (10 exercícios) }\end{array}$ & $\begin{array}{c}\text { TMR, La, FC e } \\
\text { EPOC }\end{array}$ & $22 \mathrm{~h}$ & $15 \mathrm{~min}$ & $\begin{array}{l}41 \mathrm{kcal} \mathrm{e} \\
33,5 \mathrm{kcal}\end{array}$ & $\begin{array}{l}\text { - Não utilizou repetições } \\
\text { máximas }\end{array}$ & ND \\
\hline $\begin{array}{l}\text { Melanson } \\
\text { et al, }{ }_{1}^{(18)}\end{array}$ & $\begin{array}{l}7 \mathrm{H}-\mathrm{MT} \\
(40 \pm 8)\end{array}$ & $\begin{array}{c}4 \text { séries (S) 10RM, int. } 1 \text { min (10 } \\
\text { exercícios) }\end{array}$ & $\begin{array}{l}\text { TMR, RER,VO } \\
\quad \text { e EPOC }\end{array}$ & $24 \mathrm{~h}$ & $>30 \mathrm{~min}$ & $70 \mathrm{kcal}$ & -ND duração do EPOC & $\begin{array}{l}\text { Analisar a repercussão } \\
\text { de sessão extenuante } \\
\text { de ECR sobre o tipo de } \\
\text { substrato energético } \\
\text { requerido durante } 24 \mathrm{~h} \\
\text { de análise após sessão } \\
\end{array}$ \\
\hline $\begin{array}{l}\text { Haddock e } \\
\text { Wilkin (24) }\end{array}$ & $15 \mathrm{M}-\mathrm{T}(24,2)$ & $\begin{array}{l}3 \text { séries (S) 8RM, int. 1,5 min e } 1 \text { série } \\
\text { (S) } 8 R M \text {, int. 1,5 min ( } 9 \text { exercícios) }\end{array}$ & $\begin{array}{l}\text { TMR, VO, RER, } \\
\text { La e EPOC }\end{array}$ & $120 \mathrm{~min}$ & $120 \mathrm{~min}$ & $\begin{array}{l}22,5 \pm 8 \mathrm{kcal} \mathrm{e} \\
22,3 \pm 5 \mathrm{kcal}\end{array}$ & $\begin{array}{c}\text { - Durante o EPOC não } \\
\text { houve repouso total } \\
\text { entre as medidas }\end{array}$ & ND \\
\hline Ormsbee et al. ${ }^{(28)}$ & $8 \mathrm{H}-\mathrm{T}(24 \pm 1)$ & $\begin{array}{c}3 \text { séries (C) de } 10 \text { a 85\% 10RM, int. 1,5 } \\
\text { min (6 exercícios) }\end{array}$ & $\begin{array}{l}\text { TMR, RER e } \\
\text { EPOC }\end{array}$ & $45 \mathrm{~min}$ & $45 \mathrm{~min}$ & $10 \pm 6 \mathrm{kcal}$ & $\begin{array}{l}\text { - Não analisou o } \mathrm{VO}_{2} \text { da } \\
\text { sessão }\end{array}$ & ND \\
\hline Ratamess et al. (17) & $8 H-T(21 \pm 2)$ & $\begin{array}{c}5 \text { séries (S) 75\% 1RM (10 rep), int. 0,5 } \\
\text { a } 5 \text { min e } 5 \text { séries } 85 \% 1 \mathrm{RM}(5 \text { rep), } \\
\text { int. 0,5 a } 5 \text { min (1 exercício) }\end{array}$ & $\begin{array}{c}\text { TMR, VO } \\
\text { VE, RER, } \\
\text { VE, La, FC e } \\
\text { EPOC } \\
\end{array}$ & $30 \mathrm{~min}$ & $30 \mathrm{~min}$ & \begin{tabular}{|c|}
51,8 a $44,5 \mathrm{kcal}$ \\
e 55,9 a $46,4 \mathrm{kcal}$
\end{tabular} & Apenas 1 exercício & ND \\
\hline Mazzetti et al. ${ }^{(21)}$ & $\begin{array}{c}9 \mathrm{H}-\mathrm{T} \\
(20 \pm 2)\end{array}$ & \begin{tabular}{|c|} 
4séries (S) $60 \% 1 \mathrm{RM}$, int $90 \mathrm{~s}$, \\
$2 \mathrm{~s}$ conc e exc; \\
4séries (S) $60 \% 1 \mathrm{RM}$, int $90 \mathrm{~s}, 2 \mathrm{~s}$ conc \\
e $1 \mathrm{~s}$ exc e \\
6 séries (S) $80 \% 1 \mathrm{RM}$, int $90 \mathrm{~s}, \mathrm{~s}$ conc \\
e $1 \mathrm{~s}$ exc (1 exercício) \\
\end{tabular} & $\begin{array}{c}\text { TMR, } \mathrm{VO}_{2}, \text { La e } \\
\text { EPOC }\end{array}$ & $60 \mathrm{~min}$ & $45 \mathrm{~min}$ & $\begin{array}{c}49,2 \mathrm{kcal}, \\
58,4 \mathrm{kcal} \mathrm{e} \\
39 \mathrm{kcal}\end{array}$ & Apenas 1 exercício & ND \\
\hline Farinatti et al. ${ }^{(25)}$ & $\begin{array}{l}10 \mathrm{M}-\mathrm{T} \\
(22 \pm 2)\end{array}$ & $\begin{array}{c}3 \text { séries (S) de 10RM, int. } 3 \text { min } \\
\text { (exercícios= supino, desenvolvimento } \\
\text { e tríceps) e } \\
3 \text { séries (S) de 10RM, int. } 3 \text { min } \\
\text { (exercícios = tríceps, desenvolvimento } \\
\text { e supino) }\end{array}$ & $\mathrm{VO}_{2}$ e $\mathrm{EPOC}$ & $20 \mathrm{~min}$ & $20 \mathrm{~min}$ & $\begin{array}{c}60 \pm 3 \text { kcal e } \\
57 \pm 1 \text { kcal }\end{array}$ & $\begin{array}{l}\text { - Estimou a TMR, } \\
\text { assumindo } \\
3,5 \mathrm{ml} \mathrm{O}_{2} \mathrm{~kg}^{-1} \cdot \mathrm{min}^{-1}\end{array}$ & $\begin{array}{l}\text { Apoiou-se em Mazetti } \\
\text { et al. (2007) para } \\
\text { determinar o período } \\
\text { de observação para o } \\
\text { EPOC. }\end{array}$ \\
\hline Kang et al. ${ }^{\left({ }^{30)}\right.}$ & $\begin{array}{c}11 \mathrm{H}-\mathrm{MT} \\
(21 \pm 2)\end{array}$ & $\begin{array}{c}4 \text { séries }(S) \text { à } 60 \% \text { de } 1 \text { RM, int. } 3 \text { min } \\
4 \text { séries (S) a } 75 \% \text { de } 1 \text { RM, int. } 3 \text { min } \\
4 \text { séries (S) a } 90 \% \text { de } 1 \text { RM, int. } 3 \text { min } \\
\text { (1 exercício) }\end{array}$ & $\begin{array}{l}\text { TMR, La, FC e } \\
\text { EPOC }\end{array}$ & $40 \mathrm{~min}$ & $10 \mathrm{~min}$ & $\begin{array}{c}24 \mathrm{kcal} \\
27 \mathrm{kcal} \mathrm{e} \\
14 \mathrm{kcal}\end{array}$ & $\begin{array}{l}\text { - Não revelou forma de } \\
\text { medir TMR; }\end{array}$ & ND \\
\hline
\end{tabular}

H: homens; M: mulheres; T: treinados; NT: não-treinado; MT: moderadamente treinado; (Idade): média \pm desv pad; 1RM: uma repetição máxima; RM: repetições máximas; int:. intervalo de recuperação; C: séries em circuito; S: séries consecutivas; conc.: fase concêntrica; exc.: fase excêntrica; ND: valores não declarados. 


\section{Número de séries}

Apenas dois estudos procuraram investigar especificamente o impacto do número de séries sobre o EPOC (tabela 2). Porém, um deles combinou dois métodos de ECR [série única vs trabalho em circuito] ${ }^{(24)}$ e outro manipulou, além do número de séries, o intervalo de recuperação, com o intuito de manter o mesmo volume da sessão de exercícios ${ }^{(23)}$.

Haddock e Wilkin ${ }^{(24)}$ administraram dois protocolos de ECR a muIheres jovens e treinadas, quais sejam: a) série única de oito repetições máximas (RM), com intervalo de recuperação entre séries e exercícios de 90s, em nove exercícios e duração de 21 min; b) três séries em circuito, mesma intensidade, intervalo de recuperação e número de exercícios, mas com duração de 63 min. Os autores não encontraram diferença significativa para o EPOC nas duas situações, em que pese a duração da sessão no trabalho em circuito ter sido três vezes maior (22,3 vs 22,5kcal em 60 min de duração). Com isso, concluiu-se que essa variável não influenciaria na magnitude do EPOC.

Os resultados de Melby et al. ${ }^{(23)}$ tenderam a ratificar a opinião de que o número de séries parece não influenciar o EPOC, ao menos quando se manipula apenas essa variável. Observando homens jovens e treinados em dois experimentos com séries múltiplas [seis séries de 10 exercícios com 70\% de 1RM, com intervalo de recuperação de 3 min em e duração de 90 min vs cinco séries de 10 exercícios com 70\% 1RM, com intervalo de recuperação de 4 min e duração total de 90 min], os autores verificaram que, nas duas situações, o EPOC permaneceu aumentado até o final da coleta (15h) em aproximadamente $8 \%$. A magnitude do EPOC após duas horas de coleta foi de $35 \pm 1 \mathrm{kcal}$ vs 36 $\pm 5 \mathrm{kcal}$ ou $113 \mathrm{kcal} / 15 \mathrm{~h}$ vs $114 \mathrm{kcal} / 15 \mathrm{~h}$. Esses resultados sugerem que a alteração do número de séries combinada com a manipulação do intervalo de recuperação, sem aumento na duração ou intensidade do treino, parece não influenciar significativamente o EPOC.

A interpretação da influência do número de séries a partir de estudos que não a examinaram de forma específica torna-se difícil, uma vez que é preciso considerar a interação das demais variáveis do treinamento. Assim, dos 17 estudos analisados, sete trabalharam com três séries; quatro com duas séries; dois com quatro séries; dois com seis séries; os demais usaram cinco e oito séries cada um.

Nos experimentos presentemente analisados, as intervenções foram bastante variadas, tanto em termos de intensidade quanto de volume de treinamento. De qualquer forma, analisando-se apenas os resultados finais com base em seus valores medianos e levando-se em conta as limitações dessa abordagem, nota-se que os estudos que aplicaram três séries encontraram EPOC de aproximadamente $25 \mathrm{kcal}$, para uma duração de 37,5 min. Nos estudos que utilizaram duas séries, a magnitude do EPOC foi de $35 \mathrm{kcal}$ em 40 min de duração. Os estudos com quatro, cinco, seis e oito séries apresentaram valores mais elevados, tanto para magnitude, quanto para a duração do EPOC. Entretanto, os valores medianos foram comprometidos pelo fato de haver pouca similaridade de tratamentos. Assim, os estudos que utilizaram quatro séries obtiveram $49 \mathrm{kcal}$ em $45 \mathrm{~min}$, os que aplicaram cinco séries tiveram $57 \mathrm{kcal}$ em $37 \mathrm{~min}$, os com seis séries obtiveram 76kcal em 7,6h e os com oito séries, $768 \mathrm{kcal}$ em $48 \mathrm{~h}$.

Com base nesses valores, pode-se aventar a hipótese de que protocolos de ECR com maior número de séries produziriam EPOCs com maior magnitude e duração. Todavia, vale ressaltar que dois dos três estudos que prescreveram números de séries elevadas utilizaram longos períodos para a mensuração do EPOC, o que pode ter influenciado nos valores relatados. Somada a isso, a influência de outras variáveis pode dificultar o entendimento desses dados, pois, no estudo de Ratamess et al.(17), os autores utilizaram curto intervalo de recuperação (30s) e mediram o $\mathrm{VO}_{2}$ ininterruptamente desde o término dos exercícios até o final do EPOC. Já no estudo de Dolezal et al. ${ }^{(14)}$, a medida da TMR pode ter induzido a erro, uma vez que foi realizada em condições não habituais como 12 horas de jejum. Além disso, não está claro se os sujeitos seguiram as mesmas observações determinadas para a medida controle, principalmente quanto ao nível de atividade física, dentre outros fatores que poderiam afetar a medida do EPOC ${ }^{(15,31)}$.

\section{INTERVALO DE RECUPERAÇÃO}

A influência do intervalo de recuperação sobre a fadiga muscular tem sido alvo de muitas pesquisas(32). Por outro lado, seu efeito sobre o EPOC tem sido pouco estudado. Na verdade, foram localizados apenas dois artigos que buscaram investigar o impacto da manipulação do intervalo de recuperação entre séries e exercícios sobre o EPOC (tabela 3). Haltom et al. ${ }^{(19)}$ analisaram o efeito do intervalo de recuperação (20 vs 60s) em dois protocolos envolvendo duas séries de oito exercícios realizados a 75\% de 20RM. Os resultados revelaram que a magnitude do EPOC foi significativamente maior no protocolo com menor intervalo de recuperação ( 51 vs $37 \mathrm{kcal}$ ), mas a duração em ambos os casos ficou em torno de $60 \mathrm{~min}$. Vale destacar que os autores limitaram-se a medir o EPOC por tempo determinado (60 min). Assim, pode-se cogitar que, se houvesse continuidade da medida, a magnitude e a duração do EPOC poderiam ter sido maiores.

Ratamess et al.(17) testaram diferentes intervalos de recuperação sobre o gasto calórico após o ECR em duas intensidades de treinamento ( $75 \%$ e $85 \%$ de 1 RM). Os intervalos de recuperação testados foram de 30s, 1 min, 2 min, 3 min e 5 min para cada intensidade, no exercício supino reto. A combinação de $85 \%$ de 1 RM e intervalo de 30 s produziu a maior magnitude do EPOC (55,9kcal), em comparação com os intervalos de recuperação maiores [2 min: 41,3kcal; 5 min: 46,7kcal; $p<0,05]$. A mesma tendência foi observada para o exercício realizado com 75\% de 1RM [30s: 51,8kcal; 3 min: 45,8kcal; 5 min: 44,5kcal; $p<$ $0,05]$. Ratamess et al. ${ }^{(17)}$ também utilizaram tempo fixo para o observar o EPOC (30 min), interrompendo a medida antes que seus valores retornassem à linha de base.

Logo, pouco se sabe a respeito da influência do intervalo de recu-

Tabela 2. Estudos que investigaram a influência do número de séries sobre o EPOC

\begin{tabular}{|c|c|c|c|c|c|c|}
\hline Estudo & Amostra & Protocolo & Duração/EPOC & Magnitude/EPOC & $\begin{array}{l}\text { Diferença para a } \\
\text { duração do EPOC }\end{array}$ & $\begin{array}{c}\text { Diferença para a } \\
\text { magnitude do EPOC }\end{array}$ \\
\hline Melby et al. (23) & $7 \mathrm{H}-\mathrm{T}(30 \pm 8)$ & $\begin{array}{l}\text { 1) } 6 \text { séries a } 70 \% 1 \mathrm{RM} \\
\text { 2) } 5 \text { séries a } 70 \% 1 \mathrm{RM} \\
\text { (10 exercícios) }\end{array}$ & $15 \mathrm{~h}$ & $\begin{array}{l}\text { 1) } 113 \mathrm{kcal} / 15 \mathrm{~h} \\
\text { 2) } 114 \mathrm{kcal} / 15 \mathrm{~h}\end{array}$ & NS & NS \\
\hline Haddock e Wilkin (24) & $15 \mathrm{M}-\mathrm{T}(24,2)$ & $\begin{array}{l}\text { 1) } 1 \text { série de } 8 \text { RM } \\
\text { 2) } 3 \text { séries de } 8 \text { RM } \\
\text { (9 exercícios) }\end{array}$ & $120 \mathrm{~min}$ & $\begin{array}{l}\text { 1) } 22,3 \pm 5,0 \mathrm{kcal} \\
\text { 2) } 22,5 \pm 8,0 \mathrm{kcal}\end{array}$ & NS & NS \\
\hline
\end{tabular}

H: homens; M: mulheres; T:treinados; NT: não-treinado; (Idade): média \pm desv pad; 1RM: uma repetição máxima; RM: repetições máximas; NS: sem diferença significativa entre grupos. 
Tabela 3. Estudos que investigaram a influência do intervalo de recuperação sobre o EPOC

\begin{tabular}{|c|c|c|c|c|c|c|}
\hline Estudo & Amostra & Protocolo & Duração/EPOC & Magnitude/EPOC & $\begin{array}{l}\text { Diferença para a } \\
\text { duração do EPOC }\end{array}$ & $\begin{array}{c}\text { Diferença para a magnitude } \\
\text { do EPOC }\end{array}$ \\
\hline Haltom et al. ${ }^{(19)}$ & $\begin{array}{c}7 \mathrm{H}-\mathrm{T} \\
(27 \pm 1)\end{array}$ & $\begin{array}{c}2 \text { séries, } 75 \% \text { de } 20 \mathrm{RM}, \\
\text { intervalos de } 20 \text { s e } 60 \text { s } \\
\text { (8 exercícios) }\end{array}$ & $60 \mathrm{~min}$ & $\begin{array}{l}\text { 1) } 51 \pm\left. 2 \mathrm{kcal}\right|^{*} \\
\text { 2) } 37 \pm 2 \mathrm{kcal}\end{array}$ & NS & $\begin{array}{l}42 \% \text { maior para menor } \\
\text { intervalo }\end{array}$ \\
\hline Ratamess et al. ${ }^{(17)}$ & $\begin{array}{l}8 \mathrm{H}-\mathrm{T} \\
(21 \pm 2)\end{array}$ & 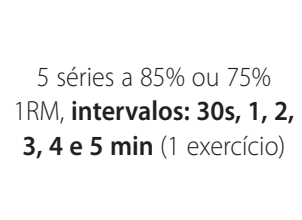 & $30 \mathrm{~min}$ & $\begin{array}{l}\text { 1) } 30 \mathrm{~s}(55,9)^{*} ; 1 \mathrm{~min}(55,8) ; \\
2 \mathrm{~min}(41,3)^{*} ; 3 \mathrm{~min}(47,3) \\
\text { e } 5 \mathrm{~min}(46,4 \mathrm{kcal})^{*} \\
\text { 2) } 30 \mathrm{~s}(51,8)^{*} ; 1 \mathrm{~min} \\
(49,1) ; 2 \mathrm{~min}(49,9) ; 3 \mathrm{~min} \\
(45,8)^{*} \text { e } 5 \mathrm{~min}(44,5 \mathrm{kcal})^{*}\end{array}$ & NS & $\begin{array}{l}\text { 1) intragupo: } 12,5 \% \text { maior } \\
\text { para menor intervalo; } \\
\text { 2) intragrupo: } 11 \% \text { maior para } \\
\text { menor intervalo; }\end{array}$ \\
\hline
\end{tabular}

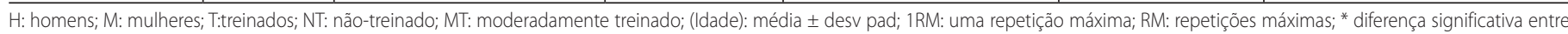
grupos; NS: sem diferença significativa entre grupos.

peração sobre a duração do EPOC. Os dois únicos estudos que investigaram a influência dessa variável de prescrição acompanharam-na por tempo fixo e determinado, provavelmente insuficiente para analisar o retorno do consumo de oxigênio à linha de base. Períodos muito breves de observação tendem a subestimar o EPOC, ao passo que longos períodos podem superestimar a medida em razão de fatores extrínsecos ao treinamento. Porém, essa informação deve ser mais bem explorada, uma vez que, dos 17 estudos que determinaram um período de observação para o EPOC, sete aferiram-no por um período de tempo equivalente ao de sua duração relatada. Ou seja, em $43 \%$ dos estudos o EPOC foi interrompido sem que os seus valores retornassem à linha de base. Nos demais, o EPOC retornou à linha de base antes do término do período determinado para a coleta.

Para analisar a influência de uma variável de prescrição sobre a duração do EPOC, deve-se considerar que a curva de recuperação do $\mathrm{VO}_{2}$ se dá de acordo com componentes relacionados a vários fatores. Esse é o caso dos substratos recompostos, como o reabastecimento dos estoques de oxiemoglobina e oximioglobina, restauração dos fosfagênios e energia necessária para a reconversão do lactato em glicogênio, que estão relacionadas ao componente rápido do $E P O C^{(11)}$. Já fatores como o aumento da temperatura corporal, manutenção de circulação hipercinética e da ventilação, estariam na origem de um EPOC elevado desde o término do exercício até a primeira hora posterior ${ }^{(13)}$. Além disso, associam-se ao componente lento ou ultralento do EPOC o aumento no metabolismo dos ácidos graxos, catecolanemia, cortisol e dano muscular, dentre outras variáveis(10,14).

Considerando a cinética do EPOC, 12 dos 17 estudos encontraram EPOC com duração menor ou igual a uma hora ${ }^{(15-17,19-22,25-28,30)}$ e apenas cinco aferiram-no durante o mesmo período de tempo para o qual relataram sua duração total(16,17,19,25,28). Ou seja, cerca de 1/3 das pesquisas apresentou a limitação metodológica de interromper a medida do EPOC antes que os seus valores retornassem à linha de base.

Quando o EPOC perdura por várias horas após o término de uma sessão, esse período é usualmente denominado de componente lento ${ }^{(13,33)}$. No caso de a TMR permanecer elevada por vários dias, fala-se em componente ultralento ${ }^{(9,10,14)}$. Dentre os estudos localizados, quatro observaram o EPOC por períodos superiores a uma hora ${ }^{(9,14,23,24)}$. Desses, aparentemente dois interromperam a medida do EPOC antes que os seus valores retornassem à linha de base ${ }^{(23,24)}$, porém dois deles não possuíam períodos de inferência padronizados ${ }^{(18,23)}$. Isso dificulta 0 entendimento dos valores relatados, pois os sujeitos ficaram expostos à influência de variáveis estranhas durante o período de observação. Os resultados desses estudos basearam-se no somatório total do gasto energético medido durante ou em alguns momentos do dia, não acompanhando a cinética do EPOC.
Quanto ao teórico componente ultralento, apenas dois estudos referiram EPOC com duração superior a 24 horas $^{(9,14)}$. Dolezal et al. ${ }^{(14)}$ investigaram a influência do ECR com ênfase no componente excêntrico da contração muscular (4s de duração) em oito séries de seis repetições máximas e 3 min de intervalo de recuperação no exercício de leg press. Partiu-se do pressuposto de que esse tipo de exercício poderia causar dano relativo ao músculo, o qual, por sua vez, necessitaria de um período importante de restauração. Assim, os autores observaram por 72 horas o impacto da sessão de ECR sobre a TMR, medindo-a em associação a dosagens enzimáticas de creatinofosfoquinase (CPK). Verificou-se que tanto a TMR quanto a CPK permaneceram elevados por até 48h, como conseqüência do desequilíbrio metabólico desencadeado pelo exercício.

Já Schuenke et al.(9), partindo da hipótese de grande quebra da homeostase, reativa à sessão de ECR desgastante e de característica hipertrófica (supercompensação) [quatro séries de 10RM em três exercícios], optaram por medir o EPOC por 48 horas. Foi encontrado EPOC elevado por até 39 horas, com magnitude de 20\% da TMR após $24 \mathrm{~h}$ e de 19\% após 39h. Ambos os estudos detectaram retorno à linha de base antes do tempo preestabelecido de observação da variável. Uma vez que esses foram os únicos trabalhos envolvendo um possível componente ultralento do EPOC em ECR, pode-se entender que o prazo máximo que se poderia esperar para a duração do EPOC estaria em torno de 48 horas.

É válido lembrar que, apesar de minoria, alguns autores ${ }^{(9,14,15,18,19,23)}$ propuseram justificativa teórica para o tempo de observação do EPOC. Em relação ao componente lento e ultralento, a justificativa teórica, na maioria dos casos, estava associada à investigação da taxa de oxidação lipídica. Ou seja, os objetivos dos estudos foram investigar o impacto do ECR sobre a TMR ou o quociente respiratório ${ }^{(18,23)}$, levando em consideração o ciclo triacilglicerol-ácidosgraxos mediado por catecolaminas. Dolezal et al. ${ }^{(14)}$ sugeriram que a lesão muscular induzida pelo ECR justificaria a aferição do o EPOC por longos períodos (72h), enquanto Schuenke et al. ${ }^{(9)}$ associaram a medida prolongada (48h) à supercompensação decorrente do treinamento hipertrófico.

Percebe-se que, de certa forma, a duração e, conseqüentemente, a magnitude do EPOC, são mais fortemente influenciadas pelo desenho experimental (forma de medir a TMR e o EPOC, inclusive períodos de observação) do que pela manipulação de uma ou mais variáveis de prescrição do ECR. A falta de informação sobre a cinética do EPOC consiste em lacuna a ser explorada, para se identificar o real impacto do ECR sobre os seus componentes.

Em que pesem as limitações aqui referidas e a fim de analisar em conjunto todos os estudos, a exemplo do que foi feito para as demais variáveis, subdividiu-se o intervalo de recuperação em três categorias: intervalo curto (20-59s) $)^{(9,15-22,24,26,28)}$, intervalo médio (60- 
$120 \mathrm{~s})^{(14,16,17,24,27,30)}$ e longo $(121-300 s)^{(14,21,23,25,27,30)}$. A mediana dos resultados encontrados nos estudos que utilizaram um intervalo curto de recuperação foi equivalente a $51 \mathrm{kcal}$ para a magnitude e 30 min para a duração do EPOC. A magnitude para a categoria de intervalo médio foi inferior para a magnitude do EPOC (39kcal), porém, mesma duração (30 min). Os estudos que adotaram longos intervalos de recuperação revelaram magnitude inferior $(46,5 \mathrm{kcal})$ àquela dos estudos com curtos intervalos de recuperação (51 kcal) e maior do que os que usaram intervalos médios (12\% maior). Já a mediana da duração dos estudos envolvendo longos intervalos de recuperação foi surpreendentemente maior (120 min). Como dito, limitações metodológicas podem estar na origem dessa disparidade de resultados.

Pode-se afirmar, então, que curtos intervalos de recuperação podem estar associados a maior impacto sobre o EPOC, principalmente em sua magnitude. Já a repercussão dessa variável sobre a duração do EPOC necessita ser mais bem investigada. Os dados sobre a influência da manipulação das variáveis de prescrição envolvendo o EPOC e o ECR ainda são escassos, não permitindo maiores conclusões.

\section{INTENSIDADE DA CARGA}

A intensidade da carga no treinamento de força, representada pelo percentual da força voluntária máxima (\%1RM) ou pelo número de repetições máximas que o indivíduo executa em cada série, é a variável que mais se destaca no cenário dos estudos sobre o EPOC, quando comparada com as demais variáveis. A tabela 4 exibe os resultados dos seis estudos que investigaram a influência específica da manipulação da intensidade do ECR sobre o EPOC.

Murphy e Schwarzkopf ${ }^{(16)}$ compararam dois diferentes protocolos de ECR com o objetivo de verificar a influência da carga mobilizada sobre o EPOC [três séries consecutivas com 80\% de 1RM, intervalo de recuperação de 2 min em seis exercícios vs três séries em circuito com 50\% de 1RM, intervalo de recuperação de 30s em seis exercícios]. É válido ressaltar que a duração total da sessão diferiu, sendo menor no trabalho em circuito. A estratégia de ECR com menor intensidade relativa e duração promoveu maior magnitude do EPOC $\left(4,9 \mathrm{~L} \mathrm{O}_{2}\right.$ vs 2,7L $\mathrm{O}_{2}$ ), enquanto sua duração foi de apenas 20 min.
Hunter et al. ${ }^{(20)}$ também trabalharam com dois protocolos [duas séries de oito repetições a $65 \%$ de 1RM, controlando a velocidade de execução em 1s para ambas as fases de contração, intervalo de recuperação de 1 min em 10 exercícios vs duas séries de oito repetições a 25\% de 1RM, com velocidade de execução de 10s para a fase concêntrica e 5s para a fase excêntrica, com mesmo intervalo entre séries e número de exercícios]. Contrariamente aos resultados de Murphy e Swartzko$\mathrm{pf}^{(16)}$, identificou-se magnitude do EPOC significativamente maior para o protocolo de maior intensidade (41,0kcal vs 33, kcal), mas duração equivalente (15 min). Esses resultados foram similares aos relatados por Thornton e Potteiger ${ }^{(26)}$, os quais encontraram EPOC de maior magnitude para o protocolo de exercício mais intenso (11,0 vs 5,5kcal), mas sem diferenças para sua duração (20 min) [duas séries de oito repetições a 85\% ou 45\% de 1RM, em nove exercícios e intervalo de $1 \mathrm{~min}]$.

Olds e Abenerthy ${ }^{(27)}$ também compararam protocolos que diferiam apenas em relação à intensidade do ECR [duas séries de sete exercícios com $75 \%$ ou $60 \%$ de 1 RM, intervalo de recuperação de 120s]. No entanto, não foi possível constatar diferença estatisticamente significativa na magnitude (41kcal vs 32kcal) e duração (60 min) do EPOC. Da mesma forma, Ratamess et al. ${ }^{(17)}$ não encontraram diferença significativa para o EPOC decorrente da manipulação da intensidade do ECR [cinco séries de um único exercício com 85\% ou 75\% de 1RM, 30s de intervalo entre séries]. Os resultados para magnitude do EPOC foram de 55,9kcal vs 51,8kcal, respectivamente, com duração de 30 min.

Kang et al. ${ }^{(30)}$ foram os únicos a relatar diferença para a duração do EPOC em função da carga, ao testar três intensidades distintas no exercício de agachamento. O EPOC com maior magnitude deu-se para o protocolo de moderada intensidade $(75 \%$ de $1 \mathrm{RM}=27 \mathrm{kcal})$, seguido por baixa intensidade ( $60 \%$ de $1 \mathrm{RM}=24 \mathrm{kcal}$ ) e alta intensidade ( $90 \%$ de $1 \mathrm{RM}=14 \mathrm{kcal}$ ). No protocolo de alta intensidade a diferença entre a magnitude do EPOC em relação à linha de base só pôde ser visualizada quando se mediu o $\mathrm{VO}_{2}$ logo após o término do exercício, desaparecendo essa diferença já no $10^{\circ}$ minuto. As sessões de menor intensidade pareceram afetar mais a duração do EPOC, com retorno do $\mathrm{VO}_{2}$ à linha de base acontecendo entre $\mathrm{O} 10^{\circ}$ e $20^{\circ}$ minutos. Os autores

Tabela 4. Estudos que investigaram a influência da intensidade da carga sobre o EPOC

\begin{tabular}{|c|c|c|c|c|c|c|}
\hline Estudo & Amostra & Protocolo & $\begin{array}{l}\text { Duração/ } \\
\text { EPOC }\end{array}$ & $\begin{array}{l}\text { Magnitude/ } \\
\text { EPOC }\end{array}$ & $\begin{array}{l}\text { Diferença para a } \\
\text { duração do EPOC }\end{array}$ & $\begin{array}{c}\text { Diferença para a } \\
\text { magnitude do EPOC }\end{array}$ \\
\hline Murphy e Schwarzkopf (16) & $10 M-T(22 \pm 2)$ & $\begin{array}{c}3 \text { séries circuito } 50 \% \text { de } 1 \mathrm{RM} \text { e } \\
3 \text { séries seguidas } 80 \% \text { de } 1 \mathrm{RM} \\
\text { (6 exercícios) }\end{array}$ & $20 \mathrm{~min}$ & $\begin{array}{l}25 \mathrm{kcal} \mathrm{e} \\
13,5 \mathrm{kcal}^{*}\end{array}$ & NS & $\begin{array}{l}96 \% \text { maior para menor } \\
\text { intensidade relativa }\end{array}$ \\
\hline Olds e Abenerthy ${ }^{(27)}$ & $7 H-\mathrm{T}(34 \pm 14)$ & $\begin{array}{c}2 \text { séries a } \mathbf{7 5} \% \text { de } 1 \mathrm{RM} \text { e } \\
2 \text { séries a } \mathbf{6 0 \%} \mathbf{1 R M} \\
\text { (7 exercícios) }\end{array}$ & $60 \mathrm{~min}$ & $\begin{array}{c}41,1 \pm 4,0 \\
\text { Kkcal e } \\
32,5 \pm 3,0 \text { kcal }\end{array}$ & NS & NS \\
\hline Thornton e Potteiger (26) & $14 \mathrm{M}-\mathrm{T}(27 \pm 5)$ & $\begin{array}{c}2 \text { séries de } 15 \text { rep a } \mathbf{4 5 \%} \text { de } 1 \text { RM } \\
2 \text { séries de } 8 \text { rep a } \mathbf{8 5 \%} \text { de } 1 \mathrm{RM} \\
\text { (9 exercícios) }\end{array}$ & $20 \mathrm{~min}$ & $\begin{array}{c}\text { 1) } 5,5 \pm 1,3 \mathrm{kcal} \\
\text { 2) } 11,0 \pm 1,9 \\
\mathrm{kcal}^{*}\end{array}$ & NS & $\begin{array}{l}97,5 \% \text { maior para maior } \\
\text { intensidade }\end{array}$ \\
\hline Hunter et al. ${ }^{(20)}$ & $7 H-T(24 \pm 3)$ & $\begin{array}{c}2 \text { séries de } 8 \text { rep a } \mathbf{6 5 \%} \text { de } \mathbf{1 R M} \text { e } \\
2 \text { séries de } 8 \text { rep a } \mathbf{2 5 \%} \text { de } 1 \mathbf{R M} \\
\text { (10 exercícios) }\end{array}$ & $15 \mathrm{~min}$ & $\begin{array}{c}41 \mathrm{kcal} \\
33,5 \mathrm{kcal} *\end{array}$ & NS & $\begin{array}{l}\text { 13,7\% maior para maior } \\
\text { intensidade }\end{array}$ \\
\hline Ratamess et al. (17) & $8 \mathrm{H}-\mathrm{T}(21 \pm 2)$ & $\begin{array}{c}5 \text { séries de } 5 \text { rep a } \mathbf{8 5 \%} \mathbf{1 R M} \text { e } \\
5 \text { séries de } 5 \text { rep a } \mathbf{7 5 \%} \mathbf{1 R M} \\
\text { (1 exercício) }\end{array}$ & $30 \mathrm{~min}$ & $\begin{array}{l}55,9 \mathrm{kcal}^{1} \\
51,8 \mathrm{kcal}^{1}\end{array}$ & NS & NS \\
\hline Kang et al. ${ }^{\left({ }^{0}\right)}$ & $\begin{array}{l}11 \mathrm{H}-\mathrm{T} \\
(21 \pm 2)\end{array}$ & $\begin{array}{c}4 \text { séries (S) a } \mathbf{6 0} \% \text { de } 1 \mathrm{RM} \\
4 \text { séries }(S) \text { a } \mathbf{7 5} \% \text { de } 1 \mathrm{RM} \text { e } \\
4 \text { séries (S) a } \mathbf{9 0 \%} \text { de } 1 \mathrm{RM} \\
\text { (1 exercício) }\end{array}$ & $\begin{array}{l}\text { TMR, La, FC } \\
\text { e EPOC }\end{array}$ & $\begin{array}{l}24 \mathrm{kcal} \\
27 \mathrm{kcal} \mathrm{e} \\
14 \mathrm{kcal}^{*}\end{array}$ & $\begin{array}{c}10 \mathrm{~min}(60 \text { e } 75 \% \text { de } \\
1 \mathrm{RM}) \text { e } \\
\mathrm{ND}(90 \% \text { de } 1 \mathrm{RM})\end{array}$ & $\begin{array}{l}90 \% \text { maior ( } 60 \text { vs } 90 \% \text { de } \\
1 \text { RM) e } \\
98 \% \text { maior ( } 75 \text { vs } 90 \% \text { de } \\
\text { 1RM) }\end{array}$ \\
\hline
\end{tabular}

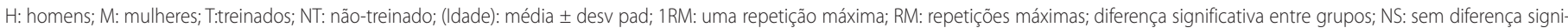
ficativa entre grupos. ' $m a i o r$ magnitude para a carga analisada (intervalo de recuperação entre séries e exercícios de 30s) 
propuseram que o impacto das estratégias de ECR sobre o EPOC seria conseqüência do volume da sessão de ECR e a intensidade de 75\% foi considerada ótima para maior EPOC. Entretanto, essa informação deve ser analisada com cautela, já que diversos estudos preconizam maior intensidade para maior EPOC $(15,17,19,20,26)$. Pode-se cogitar que, em maior número de exercícios, a cinética do EPOC poderia ser diferente, pois, com um volume de trabalho muito baixo ( $90 \%$ de $1 \mathrm{RM}$ ), o tempo de tensão muscular talvez não seja suficiente para causar respostas cardiorrespiratórias significativas. Uma indicação dessa possibilidade foi a maior concentração sanguínea de lactato nas sessões de baixa e moderada intensidades, em relação àquelas de maior intensidade (15,3 e 13,9 vs 9,7milimol).

Percebe-se, assim, que os resultados acerca da influência da intensidade do ECR sobre o EPOC são dissonantes. Dos seis estudos que se dedicaram especificamente a essa questão, dois encontraram diferença significativa para a magnitude do EPOC em sessões de maior intensidade ${ }^{(20,26)}$, dois obtiveram resultado inverso ${ }^{(16,30)}$ e outros dois não constataram diferenças entre os protocolos ${ }^{(17,27)}$. Por outro lado, apenas um estudo indicou haver relação entre a intensidade do ECR e duração do EPOC, mesmo que de forma limitada, pois o período de observação do EPOC não permitiu analisar de maneira clara sua cinética.

Certamente, disparidades metodológicas entre os estudos podem estar na origem desses resultados dissonantes. Nesse contexto, algumas considerações sobre a medida do EPOC merecem destaque. No estudo de Thornton e Potteiger ${ }^{(26)}$, a medida do EPOC iniciou-se após 20 min do final do exercício, o que pode ter subestimado os valores do gasto calórico. A medida da TMR controle com duração inferior a 30 min também pode acarretar viés, uma vez que a TMR superestimada, quando confrontada com os valores pós-exercício, pode interferir, principalmente, nos resultados referentes à duração do EPOC. Isso poderia explicar os resultados do estudo de Murphy e Schwarzkopf ${ }^{(16)}$ que mediu a TMR por apenas 5 min e Kang et al. ${ }^{(30)}$, que não descreveram os critérios para aferição da TMR. Outro problema para a comparação entre os estudos advém da ausência de padronização para a descrição dos resultados, já que nem sempre há uma estimativa do gasto calórico líquido da sessão.

Na tentativa de encontrar uma tendência geral entre todos os estudos localizados, independentemente do propósito específico de testar a influência da intensidade do ECR sobre o EPOC, os resultados disponíveis foram classificados em duas categorias: intensidade inferior ou igual a 70\% de 1RM e intensidade acima de 70\% de 1RM (tabela 1). Para os estudos agrupados na categoria inferior, as medianas da magnitude e duração do EPOC foram, respectivamente, de $41 \mathrm{kcal}$ e $45 \mathrm{~min}$. Para o grupo que trabalhou com maior intensidade, a mediana para a magnitude foi de $27 \mathrm{kcal}$ e $37 \mathrm{~min}$ para a duração. As medianas das sessões de ECR com menores intensidades, por sua vez, associaram-se a maior magnitude (64\%) e duração do EPOC (20\%).

Na verdade, não é possível apontar intensidade ótima para maior EPOC, nem que intensidades baixas e moderadas sobrepor-se-iam a altas intensidades nesse sentido ou vice-versa. Dessa forma, não foi possível encontrar justificativa fisiológica para o contraste entre a maior magnitude do EPOC em conseqüência de trabalhos com intensidades inferiores a 70\% de 1RM. Essa informação vai contra o que propõem alguns artigos de revisão ${ }^{(13,33)}$, que sugerem maior intensidade de trabalho para maior impacto sobre o EPOC.

Nota-se, ainda, que dois dos seis estudos que manipularam as intensidades detectaram respostas significativamente diversas quando uma diferença de sobrecarga de $40 \%$ foi aplicada ${ }^{(20,26)}$. Em outros três estudos ${ }^{(17,27,30)}$, quando a diferença de sobrecarga foi inferior (15\%), apenas Kang et al. ${ }^{(30)}$ relataram diferença significativa para a magnitude do EPOC. Enfim, o estudo de Murphy e Schwarzkopf $f^{(16)}$ aplicou diferença de 30\% para a sobrecarga dos grupos, tendo manipulado conjuntamente o intervalo de recuperação, não sendo possível estabelecer a influência de uma ou outra variável sobre o EPOC.

Hunter et al. ${ }^{(20)}$ aplicaram sessões com 25\% e 65\% de 1RM, encontrando EPOC com magnitude 97,5\% maior para a segunda estratégia. Thornton e Potteiger ${ }^{(26)}$ testaram protocolos de ECR com 85\% e 45\% de 1RM, também encontrando magnitude 13,7\% maior para a sessão de maior intensidade. Isso poderia sugerir, de certa forma, que para a intensidade ter impacto diferenciado sobre o EPOC, as diferenças de sobrecarga nos protocolos experimentais deveriam ser de pelo menos 30\% da força máxima voluntária. No entanto, evidentemente, essa possibilidade necessita de confirmação futura.

\section{VELOCIDADE DA CONTRAÇÃO}

A manipulação da velocidade de execução ou velocidade de contração muscular no ECR é uma das estratégias aplicadas para modificar a intensidade no treinamento da força ${ }^{(34)}$. Porém, poucos são os trabaIhos que estudaram a influência dessa variável no EPOC. Somente um estudo ${ }^{(20)}$ o fez especificamente, enquanto outros quatro divulgaram a velocidade de execução dos exercícios aplicados.

Hunter et al. ${ }^{(20)}$ compararam a resposta metabólica do ECR realizado em velocidade de contração tradicional [duas séries de oito repetições de 10 exercícios com 65\% de 1RM, velocidade de execução de um 1s para a fase concêntrica e excêntrica, além de um minuto de intervalo de recuperação] e super slow [duas séries de oito repetições com 25\% de 1RM, velocidade de execução de 10s para a fase concêntrica e 5s para a fase excêntrica, com mesmo intervalo e número de exercícios]. Verificou-se que o EPOC foi significativamente maior para a sessão com velocidade tradicional (41 kcal vs 33,5kcal em 15 min de duração). Esses valores podem ter sido motivados pela baixa intensidade no ECR feito em super slow (25\% de 1RM), o que ratificaria a possibilidade de a intensidade ser fator prevalente para maior EPOC.

Mazzetti et al. ${ }^{(21)}$ prescreveram três protocolos de ECR caracterizados pela interação entre diferentes intensidades e velocidades de execução, em sequências ditas lenta, explosiva e ultra-explosiva do exercício agachamento. Nas duas primeiras estratégias (lenta e explosiva), a intensidade foi equivalente a 60\% de 1RM, em quatro séries e 90s de intervalo de recuperação. No protocolo lento a velocidade de execução foi fixada em 2s para as fases concêntrica e excêntrica, enquanto no explosivo a velocidade programada foi de 2 s para a fase excêntrica e 1s para a concêntrica. No protocolo ultra-explosivo, a intensidade foi de $80 \%$ de 1RM, em seis séries e 90s de intervalo de recuperação, com velocidade padronizada em 2 s para a fase excêntrica e 1s para a concêntrica. Identificou-se magnitude estatisticamente diferente entre as condições lenta e explosiva até 15 min do EPOC e ultra-explosiva e rápida até 30 min do EPOC. A magnitude para 60 min de EPOC no protocolo explosivo foi de 58,4kcal, seguida do lento $(49,2 \mathrm{kcal})$ e do ultra-explosivo (39kcal). Apesar de o período de observação do EPOC ter sido delimitado em 60 min, sua duração foi de 45 min. Mazzetti et al.(21) concluíram que o protocolo explosivo, apesar de menor intensidade relativa do que o ultra-explosivo, obteve maior EPOC em virtude do maior volume da sessão. O mesmo pressuposto justificaria as diferenças entre o protocolo ultra-explosivo e lento.

Binzen et al. ${ }^{(15)}$ também utilizaram velocidades controladas em seu experimento. Os autores encontraram EPOC de $31 \mathrm{kcal}$ em $60 \mathrm{~min}$ quando o protocolo de ECR foi realizado com três séries de 10RM e velocidades de 2 s para a fase concêntrica e 4s para a excêntrica. Também utilizando $4 \mathrm{~s}$ para a fase excêntrica, Dolezal et al. ${ }^{(14)}$ verificaram que o EPOC poderia ser influenciado pela velocidade de contração. Para testar a hipótese de que o treinamento com ênfase na contração excêntrica poderia predispor a dano muscular, jovens treinados e não treinados realizaram um protocolo de ECR de alta intensidade [oito séries de 6RM] em um único exercício (leg-press), com ênfase nessa 
fase da contração. Verificou-se EPOC de longa duração (48h) e grande magnitude (768kcal) para o grupo não treinado. Dolezal et al.(14), aliás, foram os únicos autores a creditar especificamente à velocidade de execução um efeito sobre o EPOC, talvez devido a uma associação positiva entre esse tipo de treinamento e quantidade de CPK no sangue, adotada como marcadora de lesão tecidual.

\section{ORDEM DOS EXERCÍCIOS}

Dentre os elementos da prescrição do treinamento com ECR, a ordem dos exercícios tem recebido a menor atenção. Na verdade, ainda que de forma não específica, apenas Farinatti et al. ${ }^{(25)}$ publicaram dados sobre a influência da manipulação da ordem dos exercícios sobre o EPOC. Na verdade, o estudo objetivava verificar a influência da ordenação de exercícios para os membros superiores sobre o gasto calórico total da sessão, para o que foram considerados o consumo de oxigênio durante os exercícios, intervalos de recuperação entre séries de exercícios e em 20 min de EPOC. Os exercícios foram o supino reto, desenvolvimento sentado e tríceps no pulley, realizados em seqüências que iniciavam do maior para o menor grupamento muscular e viceversa [três séries de 10RM, 3 min de intervalo de recuperação]. Não houve diferença significativa em relação ao consumo total líquido de oxigênio ou para o EPOC especificamente, tendo sido o gasto calórico de $60 \pm 29 \mathrm{kcal}$ para a primeira seqüência e $57 \pm 15 \mathrm{kcal}$ para a segunda. Cabe notar, porém, que críticas podem ser feitas à adoção de tempo de observação do EPOC (limitado em 20 min), ainda que o propósito do estudo não tenha sido exatamente o de apreciá-lo.

Uma abordagem que vem sendo adotada por alguns estudos e que, de certo modo, relaciona-se (ainda que indiretamente) com a ordem dos exercícios, é a observação do efeito do modo de prescrição do treinamento da força [circuito ou séries consecutivas] sobre o EPOC. Particularmente, o trabalho em circuito tem sido muito utilizado em pesquisas com ênfase nas respostas metabólicas após uma sessão de ECR. Para autores como Burleson et al. ${ }^{(35)}$, haveria alguma semelhança entre o treinamento em circuito e sessões de exercício aeróbio, notadamente no que se refere à intensidade do trabalho.

De forma geral, acredita-se que o ECR pode favorecer a diminuição da gordura corporal e que, uma vez controlada a ingestão calórica, contribuiria com um balanço energético favorável para a manutenção ou perda de peso. De fato, são diversos os estudos que constataram que o ECR pode melhorar a oxidação lipídica, independentemente da forma de treinamento $(15,17,23,24,26,28)$. Apenas os estudos de Jamurtas et al. ${ }^{(36)}$ e Melanson et al. ${ }^{(18)}$ não confirmaram esses achados.

Sessões de ECR parecem ser compatíveis com um gasto calórico capaz de induzir alterações favoráveis na composição corporal. Silva(37), por exemplo, submetendo idosas a sessão de ECR com três séries de 10RM em três exercícios, verificou que o VO $\mathrm{O}_{2}$ médio da sessão equivalia a $50 \%$ do $\mathrm{VO}_{2 \text { máx }}$. Da mesma forma, porém utilizando o método em circuito, Burleson et al. ${ }^{(35)}$ submeteram jovens treinados a um protocolo de ECR com duas séries de oito exercícios executados com 60\% de $1 \mathrm{RM}$, encontrando $\mathrm{VO}_{2}$ médio equivalente a $44 \%$ do $\mathrm{VO}_{2 \text { máx. No que }}$ diz respeito ao gasto energético em longo prazo, Schuenke et al. ${ }^{(9)}$ investigaram o impacto do ECR prescrito na forma de circuito em jovens treinados [quatro séries, três exercícios com 10RM, intervalo de recuperação de $2 \mathrm{~min}]$. A magnitude do EPOC foi de $775 \mathrm{kcal}$ em $39 \mathrm{~h}$ de duração. Já Melanson et al.(18), valendo-se de séries consecutivas, verificaram que, em 24h, a magnitude do EPOC chegou a 70kcal. Os sujeitos executaram quatro séries de 10 exercícios com 10RM e um minuto de recuperação.

Apenas três estudos, exibidos na tabela 5, buscaram efetivamente investigar a influência do modo de prescrição sobre o EPOC, confrontando ambos os formatos. Murphy e Schwarzkop ${ }^{(16)}$ aplicaram protocolos de ECR em séries consecutivas e em circuito e verificaram que o EPOC no segundo tipo foi significativamente maior do que no primeiro, mesmo que sua intensidade tenha sido menor (25kcal vs 13,5kcal). Haltom et al. ${ }^{(19)}$ também encontraram EPOC significativamente maior em método circuito (51 kcal) do que em séries consecutivas ( $37 \mathrm{kcal})$, para uma mesma intensidade de trabalho, mas intervalos de recuperação diferentes [duas séries, 20 repetições em oito exercícios a 75\% de 20 RM, intervalo de 20s para a forma em circuito e de 60s para as séries consecutivas]. A duração da sessão também diferiu (circuito $=13 \mathrm{~min}$ e consecutiva $=23$ min).

Para controlar a limitação associada a treinamentos com duração distinta, Elliot et al. ${ }^{(22)}$ compararam sessões em circuito e com séries consecutivas pareadas pela duração (40 min) [circuito com quatro séries de 15 repetições de oito exercícios a 50\% de 1RM, com intervalo de recuperação de 30 s vs séries consecutivas com três séries de cinco repetições de oito exercícios a 85\% de 1RM, com intervalo de recuperação entre 1 e 2s min]. Não foi encontrada diferença significativa entre os grupos: no ECR em circuito a magnitude do EPOC foi de 49 kcal contra $51 \mathrm{kcal}$ no programa de séries consecutivas.

Nota-se que o método em circuito, na maioria dos estudos, esteve associado a intervalos de recuperação curtos. Assim, pode-se cogitar que a influência desse método de treinamento sobre o EPOC associarse-ia, de alguma forma, ao aumento da fadiga relativa decorrente da diminuição dos intervalos de recuperação(17). Objetivamente, quando se comparou a influência do modo de prescrição, verificou-se em dois dos três estudos que o treinamento em circuito induziria maior EPOC que o feito na forma de séries consecutivas.

Tabela 5. Estudos que investigaram a influência da forma de treinamento sobre o EPOC

\begin{tabular}{|c|c|c|c|c|c|c|}
\hline Estudo & Amostra & Protocolo & $\begin{array}{l}\text { Duração/ } \\
\text { EPOC }\end{array}$ & $\begin{array}{l}\text { Magnitude/ } \\
\text { EPOC }\end{array}$ & $\begin{array}{c}\text { Resultado } \\
\text { duração do EPOC }\end{array}$ & $\begin{array}{c}\text { Resultado Magnitude } \\
\text { do EPOC }\end{array}$ \\
\hline $\begin{array}{c}\text { Murphy e } \\
\text { Schwarzkopf (16) }\end{array}$ & $\begin{array}{l}10 \mathrm{M}-\mathrm{T} \\
(22,4)\end{array}$ & $\begin{array}{l}\text { Circuito: } 3 \text { séries } 50 \% \text { de 1RM, int. } 30 \text { s ( } 6 \text { exercícios) } \\
\text { Séries consecutivas: } 3 \text { séries a 80\% de 1RM, int. } \\
30 \text { ( } 6 \text { exercícios) }\end{array}$ & $20 \mathrm{~min}$ & $\begin{array}{l}25 \mathrm{kcal} \mathrm{e} \\
13,5 \mathrm{kcal}^{*}\end{array}$ & NS & $\begin{array}{l}96 \% \text { maior para } \\
\text { método circuito }\end{array}$ \\
\hline Haltom et al. ${ }^{(19)}$ & 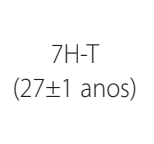 & $\begin{array}{l}\text { Circuito: } 2 \text { séries de } 20 \text { rep a } 75 \% \text { de 20RM, int. 20s } \\
\text { (8 exercícios) } \\
\text { Séries consecutivas: } 2 \text { séries de } 20 \text { rep a } 75 \% \\
\text { 20RM, int. } 60 \text { s ( } 8 \text { exercícios) }\end{array}$ & $60 \mathrm{~min}$ & $\begin{array}{l}\text { 1) } 51 \pm 2 \mathrm{kcal}^{*} \\
\text { 2) } 37 \pm 2 \mathrm{kcal}\end{array}$ & NS & $\begin{array}{l}38,5 \% \text { maior para } \\
\text { método circuito }\end{array}$ \\
\hline Elliot et al. (22) & $\begin{array}{l}4 \mathrm{H}-\mathrm{T} \text { e } 5 \mathrm{M}-\mathrm{T} \\
\text { (25,5 anos) }\end{array}$ & $\begin{array}{l}\text { Circuito: } 4 \text { séries de } 15 \text { rep a } 50 \% \text { 1RM, int. 30s } \\
\text { (8 exercícios) } \\
\text { Séries consecutivas: } 3 \text { séries } 5 \text { rep a 85\% 1RM, int. } \\
\text { 90s (8 exercícios) }\end{array}$ & $30 \mathrm{~min}$ & $\begin{array}{l}\text { 1) } 49 \mathrm{kcal} \\
\text { 2) } 51 \mathrm{kcal}\end{array}$ & NS & NS \\
\hline
\end{tabular}

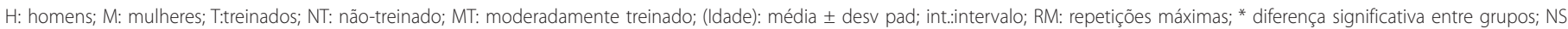
diferença não-significativa entre grupos. 


\section{CONCLUSÕES}

A análise dos estudos disponíveis sobre a influência das variáveis de prescrição do treinamento com ECR sobre o EPOC indica que ainda persistem muitas lacunas merecedoras de esforço investigativo. Em que pesem as limitações decorrentes da carência de estudos específicos, a variável cujo impacto sobre o EPOC de forma geral revelou-se mais claro foi o intervalo de recuperação entre séries e exercícios. Os dois únicos estudos que manipularam essa variável indicaram que um intervalo de recuperação curto (<60s) induziria maior magnitude do EPOC.

Por outro lado, a influência de longos intervalos de recuperação na magnitude do EPOC pareceu associar-se mais a outros aspectos do delineamento experimental, do que ao protocolo de exercício propriamente. Na verdade, verificou-se que as respostas do EPOC a longos intervalos de recuperação deram-se em estudos inespecíficos, que também se valeram de longos períodos de observação para o consumo de oxigênio após o exercício. Deve-se notar, porém, que os dois estudos sobre as relações entre EPOC e intervalo de recuperação interromperam a medida antes do retorno da TMR à linha de base. Dessa forma, pode-se afirmar que ainda não se conhece a real repercussão da manipulação dessa variável sobre a magnitude e duração do EPOC em ECR de alta intensidade. Além disso, um deles utilizou intensidades baixas e o outro. apenas um exercício, abrindo perspectivas para a investigação do papel do número de exercícios, executados com diferentes intervalos de recuperação, sobre o EPOC.

Quanto à influência da intensidade das sessões, apenas dois estudos apresentaram evidências de que cargas mais elevadas teriam maior impacto sobre a magnitude do EPOC. O efeito da manipulação da intensidade sobre sua duração ainda é incerto. O número de séries parece não exercer influência sobre o consumo de oxigênio subseqüente ao treinamento com ECR, quando se comparam protocolos de mesma intensidade e diferentes volumes. Todavia, estudos que envolveram elevados números de séries também parecem ter influenciado a mediana mais fortemente pelo seu delineamento experimental do que pela inserção dessa variável de prescrição: dos seis estudos que utilizaram quantidades elevadas de séries (> três séries), três também se valeram de períodos longos de observação do EPOC, o que pode ter influenciado nos valores obtidos.

Em relação ao modo de treinamento, velocidade de contração e ordenação dos exercícios, estudos adicionais são certamente necessários para a determinação de sua influência sobre o EPOC. No entanto, considerando as limitações apresentadas, o método em circuito pareceu associar-se a EPOCs mais intensos do que protocolos que adotaram séries consecutivas, talvez pela combinação dessa estratégia a intervalos curtos de recuperação.

Enfim, deve ser realçada a idéia de que a duração e, conseqüentemente, a magnitude do EPOC tenderam a ser fortemente influenciadas por fatores relacionados ao delineamento metodológico dos estudos (forma de medir a TMR e período de observação do EPOC). Assim, seria preciso definir critérios para subsidiar a padronização desses aspectos metodológicos, em nome de melhor comparabilidade entre resultados obtidos para o consumo de oxigênio durante e após sessões de exercícios resistidos.

Todos os autores declararam não haver qualquer potencial conflito de interesses referente a este artigo.

\section{REFERÊNCIAS BIBLIOGRÁFICAS}

1. Haskell WL, Lee IM, Pate RR, Powell KE, Blair SN, Franklin BA, et al. Physical activity and public health: updated recommendation for adults from the American College of Sports Medicine and the American Heart Association. Med Sci Sports Exerc. 2007;39(8):1423-34.

2. ACSM. American College of sports and medicine: Guidelines for Exercise Testing and Prescription. 7th Ed. Baltimore (MA): Lippincott Willians and Wilkins, 2006.

3. World Health Organization. Obesity: Preventing and managing the global epidemic. Geneva; 1998. Report of a WHO Consultation on Obesity.

4. SBC. Sociedade Brasileira de Cardiologia. IV Diretriz Brasileira Sobre Dislipidemias e Prevenção da Aterosclerose Departamento de Aterosclerose da Sociedade Brasileira de Cardiologia. Arq Bras de Cardiol. 2007, Vol 88, Suplemento

5. SBEM. Sociedade Brasileira de Endocrinologia e Metabologia. I Diretriz Brasileira de diagnóstico e tratamento da síndrome metabólica. Arq Bras Cardiol. 2005;84 (suplemento I).

6. Kraemer WJ, Volek JS, Clark KL, Gordon SE, Incledon T, Puhl SM, et al. Physiological adaptations to a weight-loss dietary regimem and exercise programs in women. J Appl Physiol. 1997;83(1):270-9.

7. Poehlman ET, Denino WF, Beckett T, Kinaman KA, Dionne IJ, Dvorak R, et al. Effects of endurance and resistance training on total daily energy expenditure in young women: a controlled randomized trial. J Clin Endocrinol Metab. 2002;87(3):1004-9.

8. Phillips WT, Ziuraitis JR. Energy cost of the ACSM single-set resistance training protocol. J Strength Cond Res. 2003;17(2):350-5.

9. Schuenke MD, Mikat RP, McBride JM. Effect of an acute period of resistance exerciseon excess post-exercise oxygen consumption: implications for body mass management. Eur J Appl Physiol. 2002;86(5):411-17.

10. Matsuura C, Meirelles CM, Gomes, PSC. Gasto energético e consumo de oxigênio pós-exercício contra-resistência. Rev Nutr Campinas. 2006;19(6):729-40.

11. Gaesser GA, Brooks GA. Metabolic bases of excess post-exercise oxygen consumption: a review. Med Sci Sports Exerc. 1984;16(1):29-43.

12. Bangsbo J, Gollnick PD, Graham TE, Juel C, Kiens B, Mizuno M, et al. Anaerobic Energy Production and O2 Defict-debt relationship during exhaustive exercise in humans. J Physiol. 1990;422:539-59.

13. Borsheim E, Bahr R. Effect of exercise intensity, duration and mode on post-exercise oxygen consumption. Sports Med. 2003;33(14):1037-60.

14. Dolezal BA, Potteiger JA, Jacobsen DJ, Benedict SH. Muscle damage and resting metabolic rate after acute resistance exercise with an eccentric overload. Med Sci Sports Exerc 2000;32(7):1202-7.

15. Binzen CA, Swan PD, Manore MM. Postexercise oxygen consumption and substrate use after resistance exercise in women. Med Sci Sports Exerc. 2001;33(6):932-8.

16. Murphy E, Schwarzkopf R. Effects of standard set and circuit weight training on excess post-exercise oxygen consumption. J Appl Sport Sci Res. 1992;6(2):88-91.

17. Ratamess NA, Falvo MJ, Mangine GT, Hoffman JR, Faigenbaum AD, Kang J. The effect of rest interval length on metabolic responses to the bench press exercise. Eur J Appl Physiol. 2007;100(1):1-17.

18. Melanson EL, Sharp TA, Seagle HM, Donahoo WT, Grunwald GK, Peters JC, et al. Twenty-four-hour metabolic responses to resistance exercise in women. J Strength Cond Res. 2005;19(1):61-6.
19. Haltom RW, Kraemer RR, Sloan RA, Hebert EP, Frank K, Tryniecki JL. Circuit weight training and its effects on excess postexercise oxygen consumption. Med Sci Sports Exerc. 1999;31(11):1613-8.

20. Hunter GR, Seelhorst D, Snyder S. Comparison of metabolic and heart rate responses to super slow vs. traditional resistance training. J Strength Cond Res. 2003;17(1):76-81.

21. Mazzetti S, Douglass M, Yocum A, Harber M. Effect of explosive versus slow contractions and exercise intensity on energy expenditure. Med Sci Sports Exerc. 2007;39(8):1291-301.

22. Elliot DL, Goldberg L, Kuel KS. Effect of resistance training on excess post-exercise oxygen consumption J Appl Sport Sci Res. 1992;6:77-81.

23. Melby C, Scholl C, Edwards G, Bullough R. Effect of acute resistance exercise on postexercise energy expenditure and resting metabolic rate. J Appl Physiol. 1993;75(4):1847-53.

24. Haddock BL, Wilkin LD. Resistance training volume and post exercise energy expenditure. Int J Sports Med. 2006;27(2):143-8.

25. Farinatti PTV, Simão R, Monteiro WD, Fleck SJ. Influence of exercise order on oxygen uptake during strength training in young women. J Strength Cond Res. 2008 (no prelo).

26. Thornton MK, Potteiger JA. Effects of resistance exercise bouts of different intensities but equal work on EPOC._Med Sci Sports Exerc. 2002;34(4):715-22.

27. Olds TS, Abernethy PJ. Postexercise oxygen consumption following heavy and light resistance exercise J Strength Cond Res. 1993;7:147-52.

28. Ormsbee MJ, Thyfault JP, Johnson EA, Kraus RM, Choi MD, Hickner RC. Fat metabolism and acute resistance exercise in trained men. J Appl Physiol. 2007;102(5):1767-72.

29. Robergs RA, Gordon T, Reynolds J, Walker TB. Energy expenditure during bench press and squat exercises. J Strength Cond Res. 2007;21(1):123-30.

30. Kang J, Hoffman JR, Im J, Spiering BA, Ratamess NA, Rundell KW, et al. Evaluation of physiological responses during recovery following three resistance exercise programs. I Strength Cond Res. 2005;19(2):305-9.

31. Schmidt WD, O'Connor PJ, Cochrane JB, Cantwell M. Restingmetabolic rate is influenced by anxiety in college men. J Appl Physiol. 1996;80:638-42.

32. Willardson JM. A brief review: factors affecting the length of the rest interval between resistance exercise sets. J Strength Cond Res. 2006;20(4):978-84.

33. Meirelles CM, Gomes, PSC. Efeitos agudos da atividade contra-resistência sobre o gasto energético: revisitando o impacto das principais variáveis. Rev Bras Med Esporte. 2004;10(2):122-30

34. Fleck SJ, Kraemer KJ. Fundamentos do treinamento de força muscular. $2^{\text {a }}$ edição. Porto Alegre: Artes Médicas, 1997

35. Burleson Jr MA, O'byant HS, Stone MH, Collins MA, Triplett-McBride T. Effect of weight training exercise and treadmill exercise on post-exercise oxygen consumption. Med Sci Sports Exerc. 1998;30(4):518-22.

36. Jamurtas AZ, Koutedakis $Y$, Paschalis $V$, Tofas $T$, Yfanti $C$, Tsiokanos $A$, et al. The effects of a single bout of exercise on resting energy expenditure and respiratory exchange ratio. Eur J Appl Physiol. 2004:92(4-5):393-8.

37. Silva NL. Variáveis do treinamento contra-resistência em idosos: dois estudos específicos. 2006. 116 f. Tese (Doutorado em Educação Física) - Universidade Gama Filho. 